\title{
Electromagnetic Shielding Woven Fabrics based on Multifilament Silver Coated with PA6,6 and PA6,6-Based Conductive Filament
}

\author{
M. Javadi Toghchi' ${ }^{1,2,3}$, C. Campagne1, C. Loghin ${ }^{2}$, I. Cristian², P.Bruniaux ${ }^{1}$, A. Cayla ${ }^{1}$, \\ Nicolae Lucano ${ }^{4}$ and Y. Chen ${ }^{3}$ \\ ${ }^{1}$ Ecole Nationale Supérieure des Arts et Industries Textiles, GEMTEX \\ Roubaix, France \\ marzieh.javadi-togh@ensait.fr \\ ${ }^{2}$ Faculty of Textile, Leather and Industrial Management, Gheorghe Asachi Technical University \\ Iasi, Romania \\ ${ }^{3}$ College of Textile and Clothing Engineering, Soochow University \\ Suzhou, China \\ ${ }^{4}$ Faculty of Telecommunication Electronics and Information Technology, Gheorghe Asachi Technical University \\ Iasi, Romania
}

\section{Extended Abstract}

Textiles have been suggested in the applications of electromagnetic shielding effectiveness (EMSE) due to the increase of the concerns about health issues caused by human exposure to the radiation [1]. A lot of products are made of metallic yarns like copper and silver for EMSE applications [2,3].

In the present study, the silver multifilament yarn $(6 \times 0.04 \mathrm{~mm})$ was coated with PA6,6 by adding an adaptive head to the extrusion process. For this purpose, PA6,6 was melted through the extrusion and formed a coating on the silver multifilament through the spinneret. The main idea was to protect silver from mechanical abrasion, oxidation, and corrosion. Also, the probable skin touch with the wearer is avoided using the coated silver with PA6,6.

The main objective of the present study was to investigate the synergism between two types of conductive yarns (coresheath as main yarn and conductive filament as supporting yarn) on EMSE of a woven structure while they either positioned parallel or perpendicular to each other. Therefore, three woven fabrics were manufactured; the first fabric was manufactured using the produced core-sheath yarn as stuffer weft in the middle of the compound woven structure (S1). It should be mentioned that the exterior layers of the woven structure were made of cotton yarns. Second fabric was weaved by adding a conductive filament of PA6,6-based nanocomposite containing multiwall carbon nanotubes and carbon black which was formerly produced using the extrusion process [5] to the compound woven structure when both the conductive yarns were overlapped. As a result, the second woven fabric was manufactured using both the core-sheath yarn and conductive filament as stuffer weft yarns while the yarn density and fabric structure remained unchanged (S2) in order to investigate the effects of adding a different kind of conductive material on EMSE. The third woven fabric was also made using core-sheath yarn in the warp direction and conductive filament in weft direction (S3).

After that, the EMSE of the samples (S1, S2, and S3) was measured in an anechoic chamber for the frequency range of 1-6 GHz [4]. EMSE of $\mathrm{S} 1$ was $25 \mathrm{~dB}$ at its maximum in the frequency band of 1-6 GHz while it was decreased by increasing the frequency from 1 to $6 \mathrm{GHz}$. Also, the maximum EMSE was $35 \mathrm{~dB}$ for S2 while the EMSE difference between S1 and $\mathrm{S} 2$ was decreased at a higher frequency $(\sim 3 \mathrm{~dB}$ at $6 \mathrm{GHz})$. The maximum level of EMSE for S3 was $28 \mathrm{~dB}$.

All three manufactured compound fabrics (S1, S2, and S3) were shielding enough to be applied in EMSE applications. However, using both conductive yarns in one direction led to higher EMSE level ( $35 \mathrm{~dB}$ vs $25 \mathrm{~dB}$ ) when other parameters like yarn density and pattern remained unchanged. The reason correlated to the amount of the conductive materials in S2 compared to S1. On the other hand, the EMSE of S3 was slightly higher than S1 (28 dB vs $25 \mathrm{~dB})$ and it was relatively low compared to S2 although the same amount of conductive yarns was used in both S2 and S3. It should be mentioned that the directive vertical antenna polarization was used in EMSE measurement in an anechoic chamber.

To conclude, the conductive yarn orientation plays an important role along with conductive materials quantity in EMSE level of the manufactured fabrics while the fabric structure was kept the same among all the samples. 


\section{References}

[1] A. Zamanian, C. Hardiman, "Electromagnetic radiation and human health: A review of sources and effects," EMR Hum. Heal., vol. 16, pp. 16-26, 2005.

[2] R. Perumalraj, B. S. Dasaradan, "Electromagnetic shielding effectiveness of doubled copper-cotton yarn woven materials," Fibres Text. East. Eur. vol. 80, pp. 74-802, 2010.

[3] H. M. Mofarah, S. S. Najar, "Investigating the electromagnetic shielding effectiveness of copper/cotton full Milano and $1 \times 1$ rib weft-knitted fabrics," J. Text. Inst., pp. 1-10, 2018 .

[4] C. Morari, I. Bălan, "Methods for determining shielding effectiveness of materials," Electro the. Electron. Autom., vol. 63, pp. 126-1362, 2015.

[5] M. Javadi Toghchi, C. Campagne, A. Cayla, P. Bruniaux, C. Loghin, I. Cristian, L. Burgnies, Y. Chen, "Electrical conductivity enhancement of hybrid pa6,6 composite containing multiwall carbon nanotube and carbon black for shielding effectiveness application in textiles," Synth. Met. ,2019. 